\title{
Real-Time Stochastic Optimum Control of Traffic Signals
}

\author{
Hee-Hyol Lee*, Member, KIICE \\ Graduate School of Information, Production, and Systems, Waseda University, Kitakyushu 808-0135, Japan
}

\begin{abstract}
Traffic congestion has become a serious problem with the recent exponential increase in the number of vehicles. In urban areas, almost all traffic congestion occurs at intersections. One of the ways to solve this problem is road expansion, but it is difficult to realize in urban areas because of the high cost and long construction period. In such cases, traffic signal control is a reasonable method for reducing traffic jams. In an actual situation, the traffic flow changes randomly and its randomness makes the control of traffic signals difficult. A prediction of traffic jams is, therefore, necessary and effective for reducing traffic jams. In addition, an autonomous distributed (stand-alone) point control of each traffic light individually is better than the wide and/or line control of traffic lights from the perspective of real-time control. This paper describes a stochastic optimum control of crossroads and multi-way traffic signals. First, a stochastic model of traffic flows and traffic jams is constructed by using a Bayesian network. Secondly, the probabilistic distributions of the traffic flows are estimated by using a cellular automaton, and then the probabilistic distributions of traffic jams are predicted. Thirdly, optimum traffic signals of crossroads and multi-way intersection are searched by using a modified particle swarm optimization algorithm to realize real-time traffic control. Finally, simulations are carried out to confirm the effectiveness of the real-time stochastic optimum control of traffic signals.
\end{abstract}

Index Terms: Traffic signal, Traffic jam, Stochastic Optimum Control, Bayesian Network, Cellular Automaton, Particle Swarm Optimization, Genetic Algorithm.

\section{INTRODUCTION}

In accordance with the increase of vehicles recently, traffic congestion has become a serious problem. In urban areas, almost all traffic congestion occurs at intersections. Intersections can be classified into three types according to the number of roads meeting at the intersection: T-junction (3-way intersection), Crossroads (4-way intersection), and Multi-way intersection (more than 4 ways). The traffic signal phases are generally more complicated at multi-way intersections and consequently traffic jams occur more easily. One of the ways to solve this problem is road expansion, but high costs and long construction periods make this option difficult in urban areas. In such cases, traffic signal control is a reasonable alternative method for reducing traffic jams.

Traffic signal controls can be classified into two types. One is an offline (pretimed) control and the other is an online (adaptive) control. In the pretimed control, Webster's formula is used to calculate the traffic signals offline using historical traffic data, but it cannot handle variation in traffic flows. On the other hand, an adaptive control can overcome this limitation by adjusting the traffic signals online in the various traffic flows. SCOOT [1], SCATS [2], and OPAC [3] have been implemented on urban traffic networks using centralized systems. However, these centralized systems require extensive data processing and computational time for calculating adequate traffic signals. In an approach using

Received 10 October 2012, Revised 05 November 2012, Accepted 22 November 2012

*Corresponding Author Hee-Hyol Lee (E-mail:hlee@waseda.jp, Tel: +81-93-692-5164)

Graduate School of Information, Production, and Systems, Waseda University, 2-7 Hibikono, Wakamatsu-ku, Kitakyushu 808-0135, Japan. 
a distributed control system, Kouvelas et al. [4] calculated adequate traffic signals according to traffic data and empirical rules. The saturated flow and traffic speed were changed according to environmental factors such as road structure and weather; therefore, the determination of appropriate parameters for the empirical rules is generally difficult. Lee et al. [5] and Dong et al. [6] calculated optimal traffic signals according to an optimal model for delay time. These methods use a mathematical traffic model to predict the future arrivals from neighborhood to intersections and to estimate the traffic delay time. However, if the road length between two intersections is very long or minor roads intersect along the road, it is difficult to precisely predict future arrivals. Recently, the various intelligence techniques such as fuzzy logic [7-11], the neural network (NN) method [12-17], and reinforcement learning (RL) method [18-23] have been used to implement distributed control systems. However, the appropriate fuzzy production rules and training data for the NN method are hard to obtain, and a long learning time is required for RL method.

This paper describes a stochastic optimum control of crossroads and multi-way traffic signals developed in the system control laboratory of Waseda University. First, a stochastic model of traffic flows and traffic jams was constructed by using a Bayesian network (BN). Secondly, the probabilistic distributions of the traffic flows are estimated by using a cellular automaton, and then the probabilistic distributions of traffic jams are predicted. Thirdly, a search for the optimum traffic signals of crossroads and multi-way intersections was performed by using a modified particle swarm optimization (PSO) algorithm to achieve real-time traffic control. Finally, simulations are carried out to confirm the effectiveness of the real-time stochastic optimum control of traffic signals.

\section{TRAFIC SIGNALS AND CONTROLS}

A traffic signal controls traffic by assigning right-of-way to one traffic movement or several non-conflicting traffic movements at a time. The right-of-way is assigned by tuning on a green signal for a certain length of time, or an interval. The right-of-way is ended by a yellow change interval during which the yellow signal is displayed, followed by the display of a red signal.

The parameters of traffic signal control, cycle, split, and offset, are typically used as illustrated in Fig.1. The cycle is a total time of signal indication of a green light, a yellow light, and a red light; the split (phase) is the green and yellow lights for right-of-way; the offset is a gap in the starting time of the green light between two traffic lights at a crossroad.

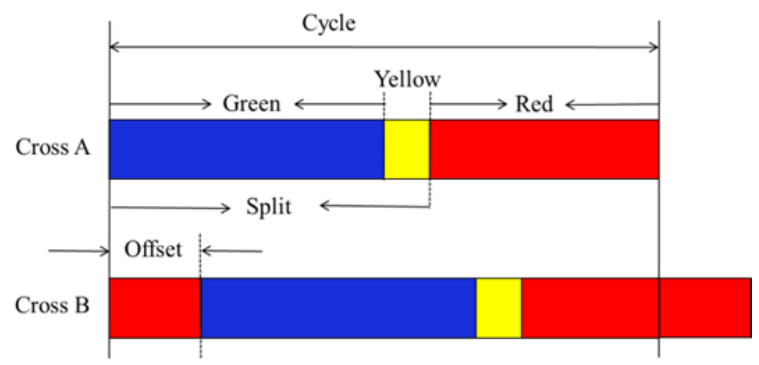

Fig. 1. Parameters of traffic signal control.

The control methods of traffic signals are classified into a point control, a line control, and a wide control from the perspective of controlled objects. Wide controls and/or the line controls generally require an enormous quantity of transmission and a great deal of time. In addition, the adjustment of the parameters for traffic signals is classified into an actuated control and a pretimed control. The trafficactuated control of isolated intersections attempts to continuously adjust green-time. These adjustments occur in accordance with real-time measures of traffic demand obtained from vehicle detectors placed on one or more of the approaches to the intersection. On the other hand, the pretimed signal control assigns right-of-way at an intersection according to a predetermined schedule. The sequence of right-of-ways (splits or phases), and the length of time interval for each signal indication in cycles is fixed based on historic traffic patterns.

In the actual situation, the traffic flow is changed randomly and its randomness makes the control of traffic signals difficult. An estimation of the traffic volume is, therefore, necessary and effective for reducing traffic jams. In addition, an autonomous distributed (stand-alone) point control for individual traffic lights is better than wide and/or line controls from the perspective of real-time control.

\section{STOCHASTIC CONTROL OF CROSSROADS TRAFFIC SIGNALS}

\section{A. Bayesian Network Stochastic Model of Traffic Flow at Crossroads}

Traffic inflows and outflows at crossroads, and resulting traffic occur randomly, and their cause-and-effect relationship can be represented by a stochastic model based on a $\mathrm{BN}$ [24].

BN is a directed and acyclic graphical model. Each node represents the variables of given objects and their direct causal relationship is represented as an arc. The relationship between each variable is evaluated quantitatively using conditional probabilities. 


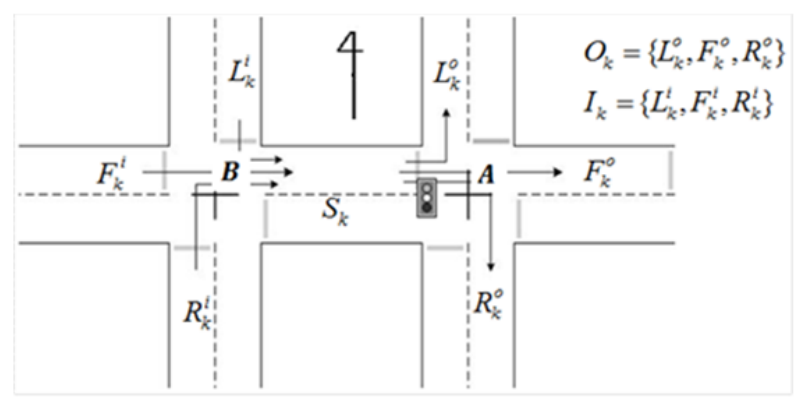

Fig. 2. Crossroads and random variables for traffic inflows, outflows, and standing vehicles.

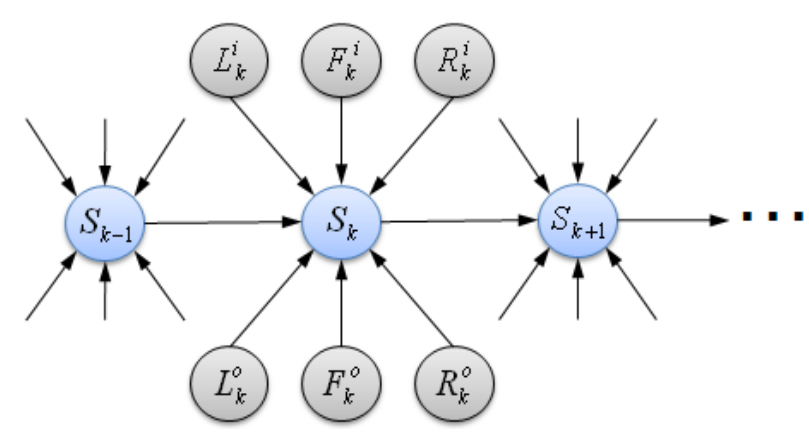

Fig. 3. The Bayesian network stochastic model of standing vehicle at crossroads.

Here, we consider two crossroads as shown in Fig. 2. The random variables of traffic inflows and outflows of the crossroad, and the standing vehicles between the two crossroads are represented as nodes. The BN model of the standing vehicles is shown in Fig. 3 [25].

The number of standing vehicles of $k$-th cycle can be calculated as Eq. (1).

$$
S_{k}=S_{k-1}+F_{k}^{o}+L_{k}^{o}+R_{k}^{o}-F_{k}^{i}-L_{k}^{i}-R_{k}^{i} .
$$

$S_{k}:$ Standing vehicles at $k$ - th cycle

$S_{k-1}:$ Standing vehicles at $(k-1)-$ th cycle

$F_{k}^{o}$ :Outflowing straight vehicles at $k$ - th cycle

$L_{k}^{o:}:$ Outflowing left turn vehicles at $k$ - th cycle

$R_{k}^{o}$ : Outflowing right turn vehicles at $k$ - th cycle

$F_{k}^{i}$ : Inflowing straight vehicles at $k$ - th cycle

$L_{k}^{i}$ : Inflowing left turn vehicles at $k$ - th cycle

$R_{k}^{i}$ : Inflowing right turn vehicles at $k$ - th cycle
The probabilistic distribution of standing vehicles $S_{k}$ at $k$ th cycle is obtained by summing over all values of the other variables as follows,

$$
\begin{aligned}
& P\left(S_{k}\right)=\sum_{S_{k-1}} \sum_{F_{k}^{i}} \sum_{R_{k}^{i}} \sum_{L_{k}^{i}} \sum_{F_{k}^{o}} \sum_{R_{k}^{o}} \sum_{L_{k}^{o}} \\
& P\left(S_{k}, S_{k-1}, F_{k}^{i}, R_{k}^{i}, L_{k}^{i}, F_{k}^{o}, R_{k}^{o}, L_{k}^{o}\right),
\end{aligned}
$$

Where

$$
\sum_{X_{i}} P\left(\Lambda, X_{i}, \Lambda\right)=\sum_{x_{i} \in \Omega_{X_{i}}} P\left(\Lambda, X_{i}=x_{i}, \Lambda\right)
$$

Following the chain rule, the joint probabilistic distribution is represented as the product of conditional probabilities as follows:

$$
\begin{aligned}
& P\left(S_{k}, S_{k-1}, F_{k}^{o}, L_{k}^{o}, R_{k}^{o}, F_{k}^{i}, L_{k}^{i}, R_{k}^{i}\right)= \\
& \quad P\left(S_{k} \mid S_{k-1}, F_{k}^{o}, L_{k}^{o}, R_{k}^{o}, F_{k}^{i}, L_{k}^{i}, R_{k}^{i}\right) \\
& \times \\
& \times\left(S_{k-1} \mid F_{k}^{o}, L_{k}^{o}, R_{k}^{o}, F_{k}^{i}, L_{k}^{i}, R_{k}^{i}\right) \\
& \times
\end{aligned}
$$

According to the D-separation [24] and the standing vehicle $S_{k}$ at $k$-th cycle determined by Eq. (1), Eq. (2) can be represented as,

$$
\begin{aligned}
& P\left(S_{k}\right)= \\
& \sum_{S_{k-1}} \sum_{F_{k}^{o}} \sum_{L_{k}^{o}} \sum_{R_{k}^{o}} \sum_{F_{k}^{i}} \sum_{L_{k}^{i}} \sum_{R_{k}^{i}} P\left(S_{k-1}\right) P\left(F_{k}^{o}\right) P\left(L_{k}^{o}\right) P\left(R_{k}^{o}\right) \\
& P\left(F_{k}^{i}\right) P\left(L_{k}^{i}\right) P\left(R_{k}^{i}\right)
\end{aligned}
$$

However, the probabilistic distributions of the outflows $P\left(F^{o}{ }_{k}\right), P\left(L^{o}{ }_{k}\right), P\left(R^{o}{ }_{k}\right)$ and the inflows $P\left(F_{k}^{i}\right), P\left(L_{k}^{i}\right), P\left(R_{k}^{i}\right)$ are necessary to calculate the probabilistic distribution $P\left(S_{k}\right)$ of the standing vehicles because we are now located at the point on the time axis illustrated in Fig. 4.

The probabilistic distributions of traffic outflows and inflows at $k$-th cycle are estimated by their updating. The altered traffic signals will change the probabilities of the outflowing and inflowing vehicles. To treat this situation, two patterns are considered here.

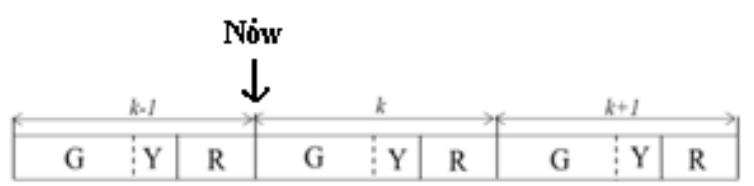

Fig. 4. Position for prediction of probabilities. G: green light, Y: yellow light, $R$ : red light. 


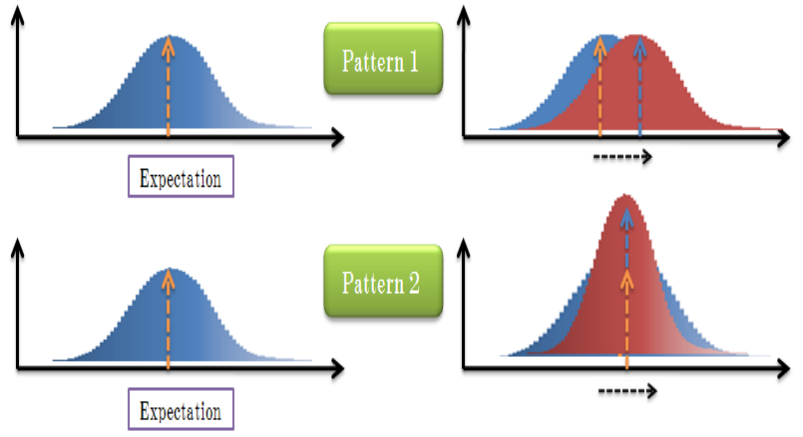

Fig. 5. Updating of probabilistic distribution for traffic flow.

Pattern 1 is a shifting of the probabilistic distributions according to the fluctuation of green-time, and pattern 2 is an increase in the probability for neighboring expectation and a decrease of others (Fig. 5). Pattern 2 is adopted to update the probabilistic distributions of traffic flows based on simulations.

In order to deduce adequate traffic signals, the probabilistic distributions of standing vehicles are predicted to three cycles ahead as an example in this paper. The procedure of updating for prior probabilities and prediction for probabilistic distributions of standing vehicles is illustrated in Fig. 6. First, the prior probabilities of each variable are updated by previous data at cycle $(k-1)$. Then, the probabilistic distributions of standing vehicles at $k$-th cycle are predicted using Eq. (4). Next, a rule-based algorithm, which will be described in the next section, is applied to deduce adequate traffic signals and it is used for updating the prior probability of the outflow. Finally, the probabilistic distribution of standing vehicles at $k$-th cycle is calculated to predict the following cycles. The procedures of prediction at cycles $(k+1)$ and $(k+2)$ are the same as at $k$-th cycle.

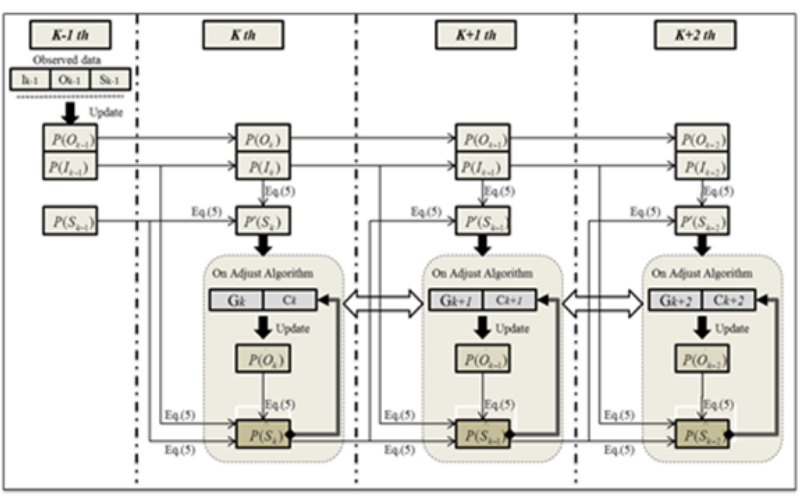

Fig. 6. Procedure of updating for prior probabilities and prediction for standing vehicles.

\section{B. Traffic Signal Control Based on Predicted Probabilistic Distributions of Standing Vehicles}

\section{1) Rule-Based Control}

Reduction of traffic jams for a major street (e.g., the eastwest direction of Fig. 2) can be achieved by extension of green-time to reduce the probabilistic distribution of the major street. However, in this case, the probability of traffic jams for a minor street (for example, the north-south direction) will be increased by the long red time of the minor street. Therefore, the probabilities of an over-standing vehicle $S_{\max }$ (i.e., traffic jam) and an under-standing vehicle $S_{\text {min }}$ for the major street must be reduced all together for the reduction of traffic jams at crossroads as shown in Fig. 7.

According to the BN stochastic model, the probabilistic distribution of the standing vehicle, splits, and cycle time of traffic signals are controlled by using the predicted probabilistic distributions of standing vehicles. A rule-based control procedure is as follows:

Step 1: Predict probabilistic distribution of standing vehicles by using a BN stochastic model to three cycles ahead.

Step 2: Calculate probabilities $S_{\max }$ or above and $S_{\min }$ or below of standing vehicles.

Step 3: Compare these probabilities with the desired values.

Step 4: Adjust split and cycle time until probabilities $S_{\max }$ or above and $S_{\min }$ or below satisfy the desired values.

The flowchart of the procedure for traffic signal control is shown in Fig. 8.

\section{2) Back Propagation NN Control}

Traffic signal control is composed of two procedures: online and offline processing. In the online processing, a back propagation neural network (BPNN) is used to calculate adequate traffic signals based on the result of a BN stochastic model for the prediction of standing vehicles. Then, the rule-based algorithm is applied to update the weight of the BPNN model in the offline processing.

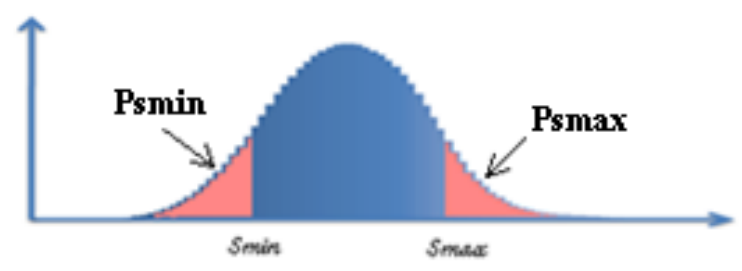

Fig. 7. Probabilistic distribution of standing vehicle for major street. 


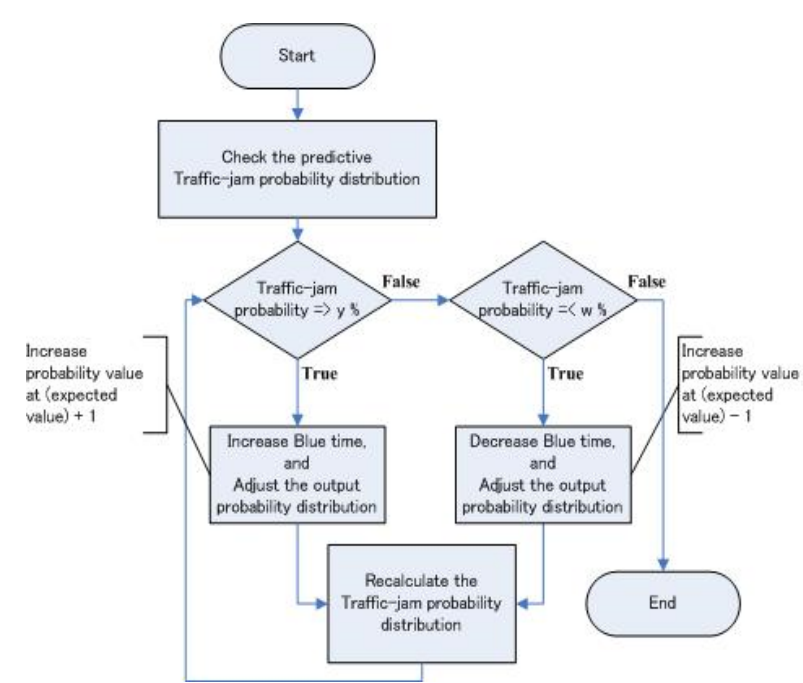

Fig. 8. Flowchart of rule-based control.

The BPNN model has a powerful learning capability, and this model consists of an input layer, a hidden layer, and an output layer. The BPNN model for traffic signal control is shown in Fig. 9 [26]. The input values are the probabilities of standing vehicles, which are predicted by the BN model, and the output values are traffic signals.

In this BPNN model, the number of neurons for the input layer is 141, and the inputs are the probabilities of standing vehicles. The traffic signal comprises two parameters: split time and cycle length. Therefore, two neurons in the output layer are set up. The number of the hidden layer can be calculated by an empirical formula Eq. (5), where $n, m$, and $l$ are the numbers of neurons in the input layer, hidden layer, and output layer, respectively.

$$
m=\sqrt{n \times l}
$$

The procedure of traffic signal control using the BPNN model is illustrated in Fig. 10.

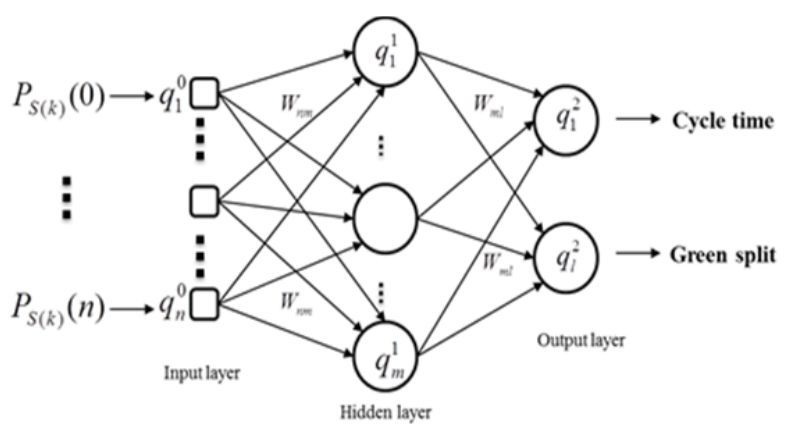

Fig. 9. Structure of back propagation neural network model.

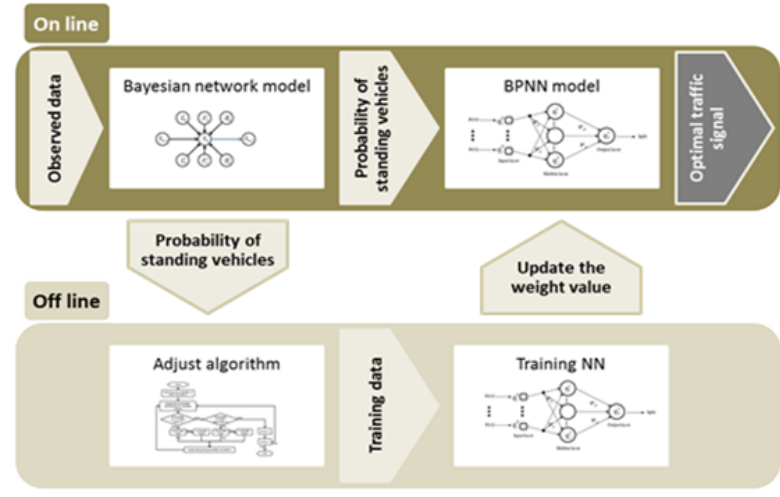

Fig. 10. Procedure of traffic signal control using back propagation neural network (BPNN) model.

\section{Stochastic Optimum Control of Crossroads Traffic Signals}

\section{1) Formulation for Optimization of Traffic Congestion Problem}

It is necessary to reduce traffic jams of a major street and a minor street both for the reduction of traffic volume at crossroads. However, when the green-time of the major street is extended to reduce the traffic jams of the major street, the traffic congestion of the minor street will be increased because the red-time of the minor street has been extended. Therefore, the traffic congestion problem at crossroads is a trade-off between the length of green-time and red-time (i.e., green-time of the minor street) for the major street. An adequate method of the reduction of traffic volume at crossroads is a prediction of probabilistic distributions of traffic jams and reduction of probabilities for over-standing vehicles and under-standing vehicles of the major street together.

As an optimization problem of traffic congestion at crossroads, a performance criterion is defined as follows [25]:

$$
\begin{aligned}
J_{m}= & w_{1} \cdot \sum_{S_{k}=0}^{S_{k}=S_{\min }} \hat{P}^{t_{m}^{i}}\left(S_{k}\right) \\
& +w_{2} \cdot \sum_{S_{k}=S_{\max }}^{S_{k}=+\infty} \hat{P}^{t_{m}^{i}}\left(S_{k}\right),
\end{aligned}
$$

where $\hat{P}^{t_{m}^{i}}\left(S_{k}\right)$ is a predicted probabilistic distribution of the major street when the traffic signals are $t_{m}^{i}$ at the $m$-th search, and weight coefficients $w_{1}$ and $w_{2}$ are set by the levels of importance for the major street and minor street as shown in Fig. 7.

\section{2) Construction of Traffic Flow Micro-Simulator by Cellular Automaton}

The probabilistic distribution of traffic outflows is necessary to predict the probabilistic distribution of standing vehicles at $k$-th cycle. 


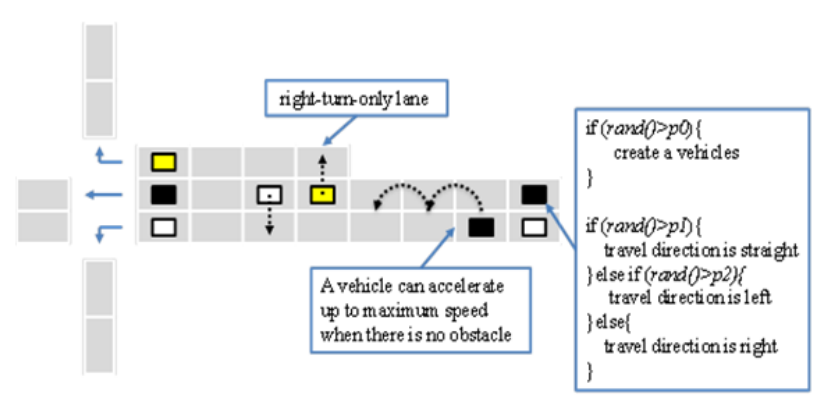

Fig. 11. Rules of vehicle movements in the micro-simulator.

The traffic outflows are estimated by using an urban traffic flow micro-simulator based on a cellular automaton (CA), which is an example of artificial life, built based on a model of a highway [27]. The rules of vehicles movements on roads are illustrated in Fig. 11, where the parameters $p 0$, $p 1$, and $p 2$ are determined by measured data.

Input to cell: According to the generation of a random number for an inflow rate, a vehicle is generated and the direction of travel to the next intersection is determined. The inflow rate of each road is determined by measured traffic data.

Movements: A vehicle can accelerate up to a maximum speed when a front cell is free of obstruction. Considering a mean speed in an urban area, the maximum speed is set at 2 cells $/ 1$ step $(1$ cell $=7.5 \mathrm{~m} ; 1$ step $=1$ $\mathrm{sec})$. According to the density of the traffic, the speed can change randomly. On multi-lane roads, vehicles can move in parallel lanes. When a vehicle turns right, the vehicle moves to a right-turn-only lane. (Following Japan's traffic patterns, the model assumes driving on the left side of the street.)

Intersection: When a traffic signal is green, a vehicle is allowed to cross the intersection according to its direction of travel, and the direction of travel at the next intersection is reset.

\section{3) Searching for Traffic Signals by Using the PSO Method}

The PSO method was introduced by Kennedy and Eberhart [28]. The PSO method is based on a simple mechanism, which mimics the swarming behavior in birds flocking and fish schooling to guide particles to search for optimal solutions. The PSO method is easy to implement and use with only a few parameters to adjust. In the PSO method, a swarm of particles is represented as potential solutions and each particle is associated with a velocity vector $v^{m}$ and a position vector $x^{m}$.

During the evolutionary process, the velocity and position of a particle $i$ on search number $m$ are updated by Eqs. (7) and (8).

$$
\begin{gathered}
v_{i}^{m+1}=w \cdot v_{i}^{m}+c_{1} \cdot \operatorname{rand}() \cdot\left(\text { pbest }_{i}^{m}-x_{i}^{m}\right) \\
+c_{2} \cdot \operatorname{rand}() \cdot\left(\text { gbest }^{m}-x_{i}^{m}\right) \\
x_{i}^{m+1}=x_{i}^{m}+v_{i}^{m+1}
\end{gathered}
$$

where $w$ is an inertia weight, $c_{1}$ and $c_{2}$ are acceleration coefficients, and rand ( ) is a random number within [0,1]. In Eq. (7), pbest is the best position of a particle, and gbest is the best position of all particles with the best fitness during the evolutionary process, and then an optimum point is found by updating the positions and velocities of the particles as illustrated in Fig. 12. The structure of the stochastic optimum control for traffic jams at crossroads is shown in Fig. 13. The calculation procedure is as follows:

Step 1: Set the number of particles in the swarm, and an initial position and velocity of each particle. The initial position of a particle $i: \boldsymbol{t}_{o}^{i}=\left(g_{o}^{i}, y_{o}^{i}, r_{o}^{i}\right)$ represents the signal times of green, yellow, and red, respectively, and their initial times are produced randomly. Also, the initial velocity of the particle $i: v_{o}^{i}=\left(v_{g o}^{i}, v_{y o}^{i}, v_{r_{o}}^{i}\right)$ represents the initial updating of green, yellow, and red times, and their initial number of updates are produced randomly in $[-2,+2]$. Set the number of searches to $m=0$.

Step 2: Estimate the traffic inflows $\hat{I}_{k}$ and outflow $\hat{O}_{k}$ of crossroads at $k$-th cycle by using the CA micro-simulator.

Step 3: Update the prior probabilistic distributions of traffic inflow $\hat{P}\left(I_{k}\right)$ and outflow $\hat{P}\left(O_{k}\right)$ at $k$-th cycle using the measured data at cycle $(k-1)$ and the estimated $\hat{I}_{k}$ and $\hat{O}_{k}$.

Step 4: Predict the probabilistic distribution of traffic jam $\hat{P}\left(S_{k}\right)$ at $k$-th cycle using the BN stochastic model.

Step 5: Calculate the performance criterion $J_{m}$ using $\widehat{P}\left(S_{k}\right)$.

Step 6: Update pbest and gbest of the PSO algorithm.

Step 7: If $m<m_{\max }$ : update the positions and velocities of all particles, and Go to step 2;

Else, output the positions of gbest (green - yellow - red times).

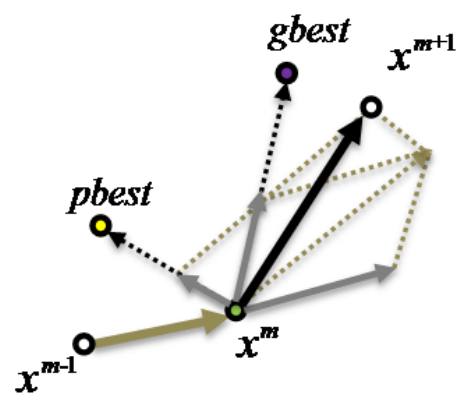

Fig. 12. Particle swarm optimization algorithm. 


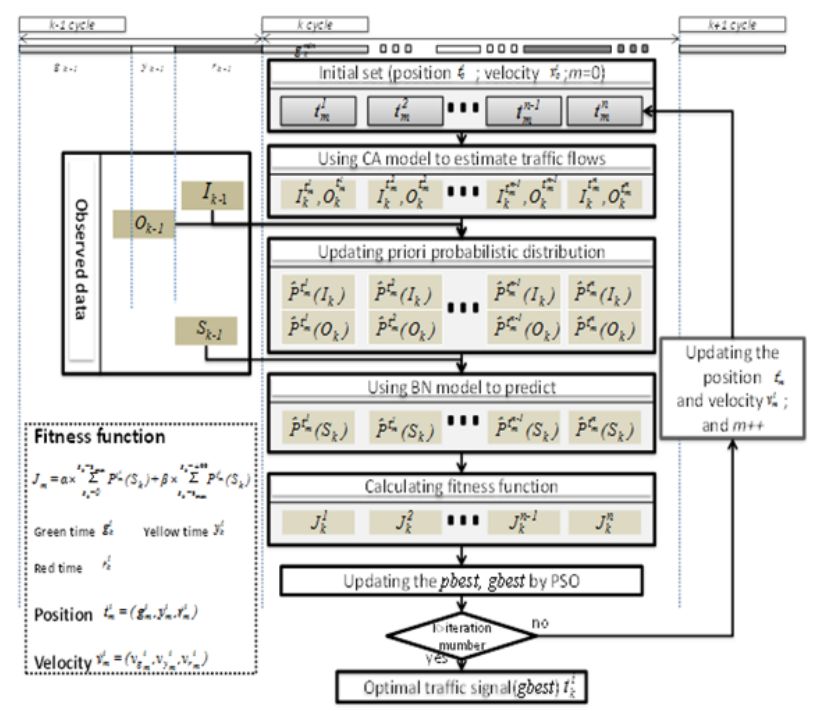

Fig. 13. Structure of traffic signal control based on predicted probabilities of traffic jams. BN: Bayesian network, CA: cellular automaton, PSO: particle swarm optimization.

\section{Simulation}

A simulation was carried out to confirm the effectiveness of stochastic control of traffic signals at crossroads using actual traffic flow data. The actual data was measured on 17 January 2008, 5:00-7:00 PM at Tutuimachi crossroads, Kitakyushu, Japan, as illustrated in Fig. 14. The traffic light of the Tutuimachi crossroads is a pretimed control, its cycle length is 150 seconds and 4 phases, and their traffic lighting times are shown in Table 1.

In addition, the set values are $S_{\min }=30(\mathrm{pcu}), S_{\max }=60$ (pcu); the probabilities equal or less than $S_{\min }$ and more than $S_{\text {max }}$ are $P_{\text {min }}=P_{\text {max }}=0.15 ; w_{1}=0.3, w_{2}=0.7$ in Fig. 7 and Eq. (6), respectively.

The predicted probabilistic distribution of traffic jams four cycles ahead, and the prior probabilistic distributions at Cycle 3 and the updated one at Cycle 4 of traffic inflows and outflows are shown in Figs. 15 and 16, respectively.
Table 1. Traffic signals of pretimed control and their adjustable range

\begin{tabular}{c|c|c|c|c}
\hline & Phase 1 & Phase 2 & Phase 3 & Phase 4 \\
\cline { 2 - 5 } & \multicolumn{1}{|c}{} & 18 & 36 & 18 \\
\hline $\begin{array}{c}\text { Pretimed } \\
\text { range }\end{array}$ & 78 & $10-25$ & $30-60$ & $10-25$ \\
\hline
\end{tabular}

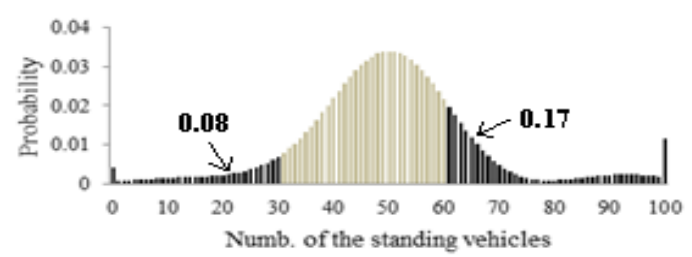

(a)

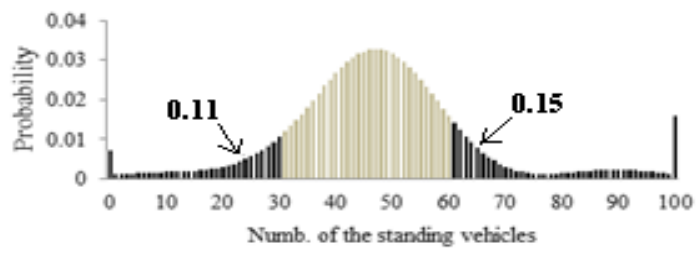

(b)

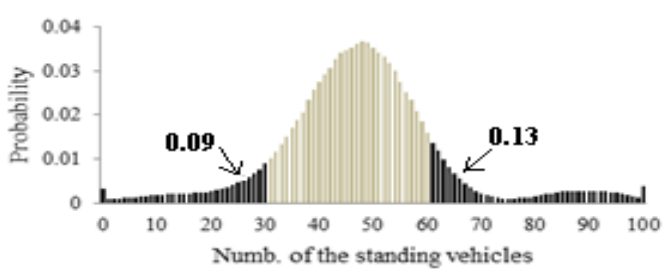

(c)

Fig. 15. Probabilistic distributions of standing vehicles. (a) Pretimed control, (b) rule-based control, and (c) particle swarm optimization control.

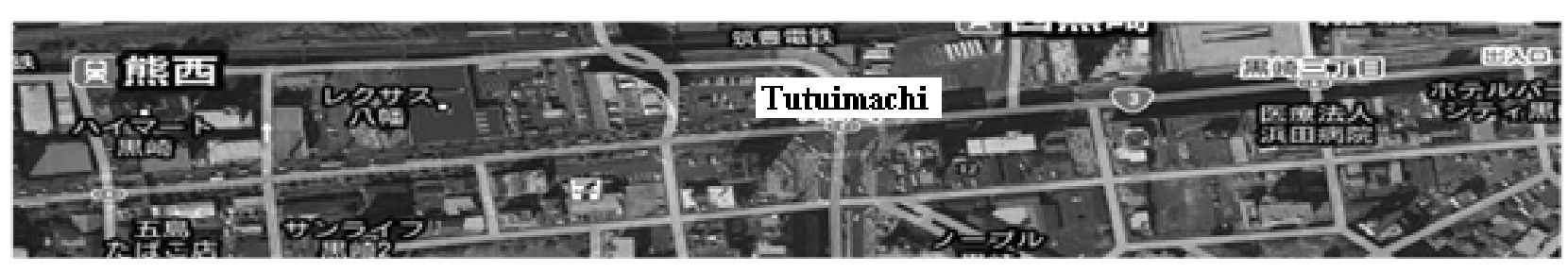

Fig. 14. Map of Tutuimachi crossroads. 

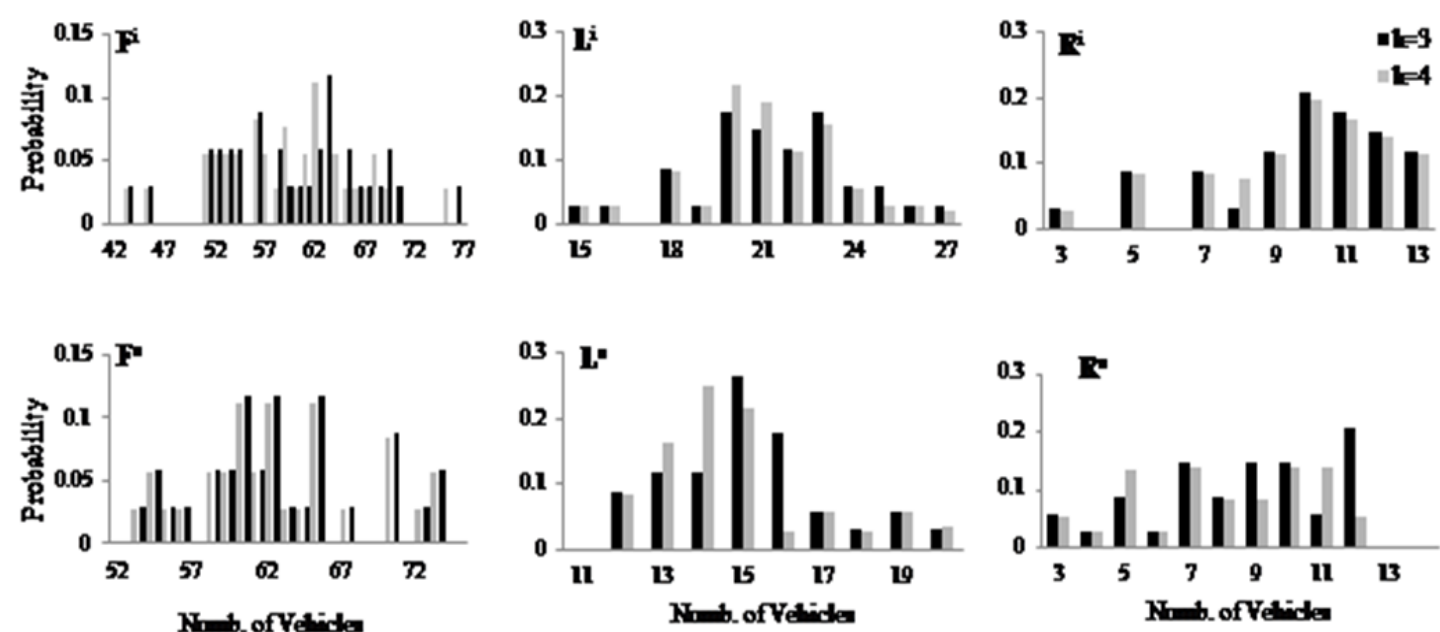

Fig. 16. Prior and updated probabilistic distributions of inflows and outflows.

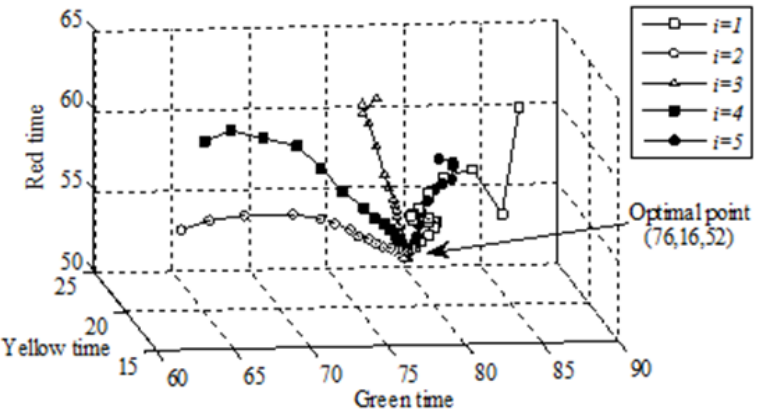

Fig. 17. Convergence of 5 particles at Cycle 4.

Moreover, the convergence of 5 particles (of the total 30 particles) at Cycle 4 is illustrated in Fig. 17, where the initial positions $\left(g_{m}{ }^{i}=86, y_{m}{ }^{i}=21, r_{m}{ }^{i}=61 ; i=1\right)$ converged into an optimum point $(76,16,52)$, and the convergences of fitness values for pbest and gbest are illustrated in Fig. 18.

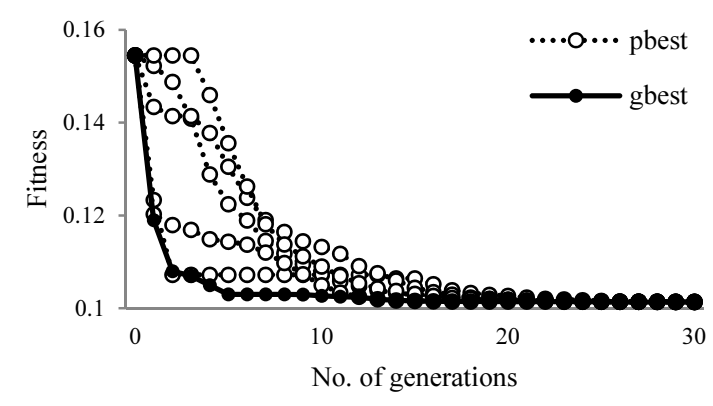

Fig. 18. Fitness values by particle swarm optimization control

The traffic signals by pretimed, rule-based, and PSO controls and the traffic jams at crossroads are shown in Figs. 19 and 20 , respectively.

The number of standing vehicles on the major street and the total number of PSO controls was reduced by $12(\%)$ and $8(\%)$ compared with the pretimed control, respectively.
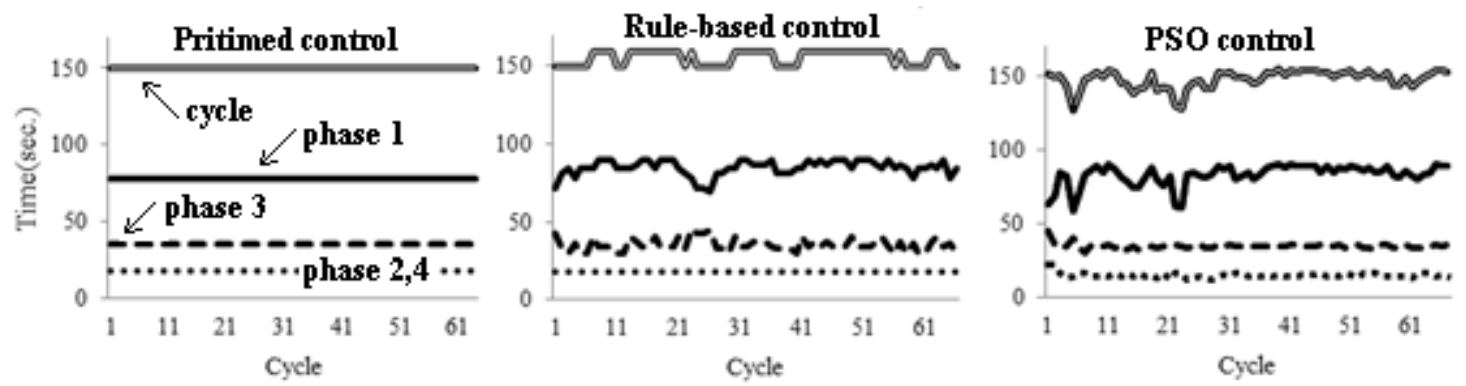

Fig. 19. Comparison of traffic signals. 


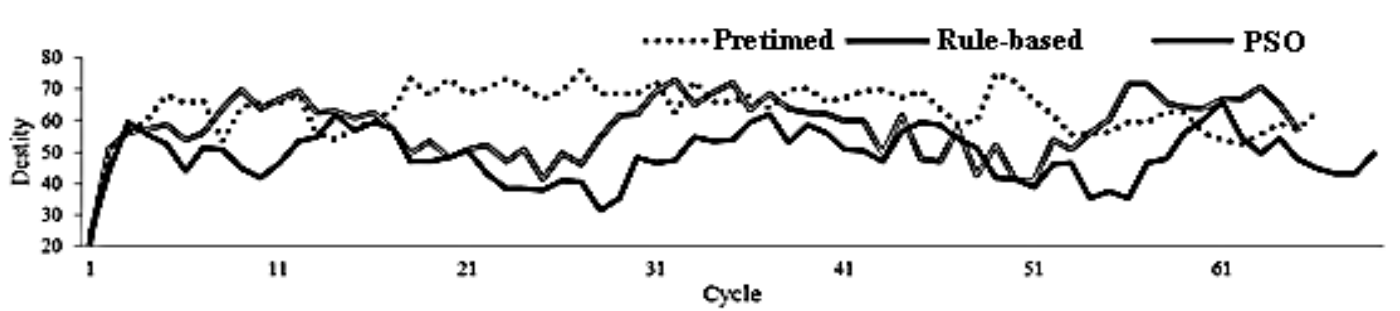

(a)

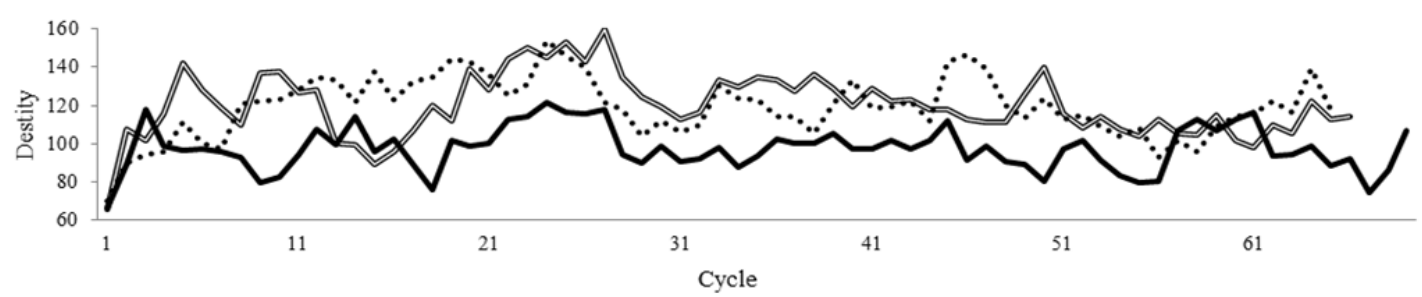

(b)

Fig. 20. Traffic jams of crossroads. (a) Number of standing vehicles on the major street (east-west direction), (b) total number of standing vehicles.

\section{REAL-TIME STOCHASTIC OPTIMUM CONTROL OF MULTI-WAY TRAFFIC SIGNALS}

\section{A. Construction of BN Stochastic Model for Traffic Flow at Multi-Way Intersection}

Here, we consider an $r$-way intersection illustrated in Fig. 21. At a road $h$, the traffic inflows, outflows, and standing vehicles are represented as Eq. (9).

$$
S_{k}^{h}=S_{k-1}^{h}+I R_{k}^{h}+I F_{k}^{h}+I L_{k}^{h}-\sum_{j=1 ; j \neq h}^{r} O_{k}^{j}
$$

On the $r$-way intersection, the casual relationship between traffic inflows, outflows, and standing vehicles can be represented by the BN model illustrated in Fig. 22. The probabilistic distribution of standing vehicles at $k$-th cycle is obtained by summing over all values of the other variables as follows:

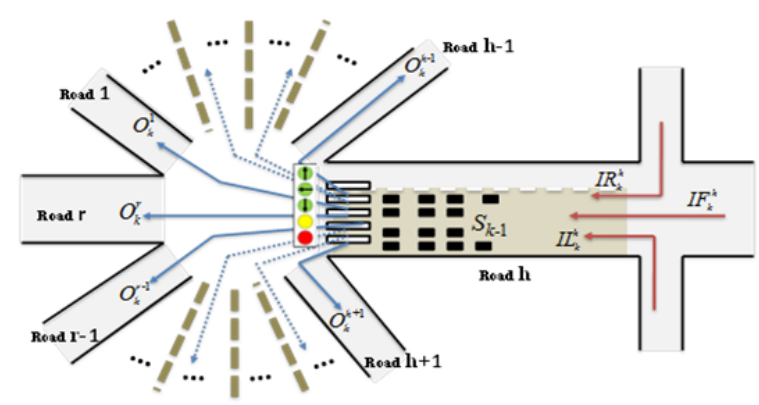

Fig. 21. The r-way intersection.

$$
\begin{aligned}
& P\left(S_{k}^{h}\right)=\sum_{S_{k-1}^{h} I R_{k}^{h} I F_{k}^{h}} \sum_{I L_{k}^{h}} \sum_{O_{k}^{1}} \sum_{O_{k}^{2}} \Lambda \sum_{O_{k}^{h-1} O_{k}^{h+1}} \sum_{O_{k}^{r-1} O_{k}^{r}} \sum \\
& P\left(S_{k}^{h}, S_{k-1}^{h}, I R_{k}^{h}, I F_{k}^{h}, I L_{k}^{h}, O_{k}^{1}, O_{k}^{2}, \Lambda, O_{k}^{h-1}, O_{k}^{h+1}, \Lambda, O_{k}^{r-1}, O_{k}^{r}\right)
\end{aligned}
$$

In consideration of

$$
P\left(S_{k}^{h} \mid S_{k-1}^{h}, I R_{k}^{h}, I F_{k}^{h}, I L_{k}^{h}, O_{k}^{1}, O_{k}^{2}, \ldots, O_{k}^{r}\right)=1,
$$

with the chain rule and D-separation, Eq. (10) can be represented as

$$
\begin{aligned}
P\left(S_{k}^{h}\right)= & \sum_{S_{k-1}^{h}} \sum_{I R_{k}^{h}} \sum_{I F_{k}^{h}} \sum_{I L_{k}^{h}} \sum \sum_{O_{k}^{1}} \Lambda \sum_{O_{k}^{2}} \sum_{O_{k}^{h-1}} \Lambda \sum_{O_{k}^{h+1}} \sum_{O_{k}^{n-1}} \\
& P\left(S_{k-1}^{h}\right) P\left(I R_{k}^{h}\right) P\left(I F_{k}^{h}\right) P\left(I L_{k}^{h}\right) P\left(O_{k}^{1}\right) \\
& \times \Lambda P\left(O_{k}^{h-1}\right) P\left(O_{k}^{h+1}\right) \Lambda P\left(O_{k}^{r}\right)
\end{aligned}
$$

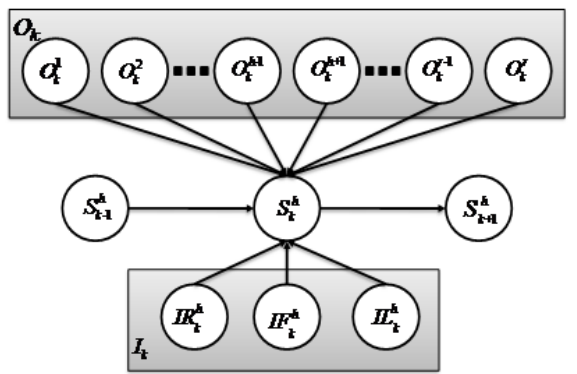

Fig. 22. The Bayesian network stochastic model of r-way intersection. 


\section{B. Real-Time Stochastic Optimum Control of $r$-Way Traffic Signals}

\section{1) Formulation for Optimization of Traffic Congestion Problem at $r$-Way Intersection}

For the $r$-way intersection, the situation of traffic jams is regarded as that the standing vehicles for all roads are bigger than $S_{\text {max }}^{h}(h=1,2, \ldots, r)$ in this paper. Therefore, the optimization problem of traffic congestion at $r$-way intersections is formulated as follows [29]:

$$
\begin{gathered}
F\left(t^{1}, t^{2} \ldots t^{n-1}, t^{n}\right)=\sum_{h=1}^{h=r} w^{h} \cdot\left[\sum_{S_{k}^{h}=S_{\max }^{h}}^{+\infty} P\left(S_{k}^{h}\right)\right] \rightarrow \min \\
\text { subject to } t_{\min }^{i} \leq t^{i} \leq t_{\max }^{i}, \quad i=1,2, \ldots, n .
\end{gathered}
$$

where $i$ is the number of $n$ signal phases, $h \quad(h=1,2, \ldots, r)$ is the number of roads, $k$ is the cycle number, $t^{i}$ is the time length of the signal phase $i, t_{\min }^{i}$ and $t_{\min }^{i}$ are an adjustable range of the signal phase $i, w^{h}$ is a weight coefficient, $S_{\max }^{k}$ is a set value for traffic jams, and $P\left(S_{k}^{h}\right)$ is a predicted probabilistic distribution of road $h$ at $k$-th cycle when the traffic signals are $\left(t^{1}, t^{2}, \Lambda t^{n-1}, t^{n}\right)$, respectively.

\section{2) Traffic Flow Micro-Simulator by CA Model for r-Way Intersections}

In order to predict the probabilistic distributions of standing vehicles, an updating rule for the prior probabilities of traffic inflows is indispensable. In this paper, Eq. (14) is used to update the prior probabilistic distributions as follows:

$$
\begin{aligned}
& \hat{P}\left(I_{k}^{j}\right):=P\left(I_{k-1}^{j}\right)-\frac{\gamma}{\eta-1} \quad\left(I_{k-1}^{j} \neq \hat{I}_{k}\right) \\
& \hat{P}\left(I_{k}^{j}\right):=P\left(I_{k-1}^{j}\right)+\frac{\eta \gamma}{\eta-1} \quad\left(I_{k-1}^{j}=\hat{I}_{k}\right) \\
& \hat{P}\left(O_{k}^{j}\right):=P\left(O_{k-1}^{j}\right)-\frac{\gamma}{\eta-1} \quad\left(O_{k-1}^{j} \neq \hat{O}_{k}\right) \\
& \hat{P}\left(O_{k}^{j}\right):=P\left(O_{k-1}^{j}\right)+\frac{\eta \gamma}{\eta-1} \quad\left(O_{k-1}^{j}=\hat{O}_{k}\right) \quad j \in(0,1,2, \ldots, \eta)
\end{aligned}
$$

where $j$ is the number of events for traffic flows at $(k-1)$ cycle, $\eta$ is the number of total events, $\gamma$ is an empirical value, and $\hat{I}_{k}$ and $\hat{O}_{k}$ are the predicted traffic flows using the modified CA traffic micro-simulator of the $r$-way intersection.

An example illustrating the rules of movements for vehicles on a road network is shown in Fig. 23.

Input to cell: A vehicle is generated and its direction of travel on the intersection is determined according to the comparison of a random number $[0,1]$ with a set value.

Speed: A vehicle is accelerated up to a maximum speed, where maximum speed $=2$ moves $/$ cell; 1 cell $=7.5 \mathrm{~m} / 1$ step ( 1 second), when a front cell is free of obstruction. The speed is changes randomly according to the road conditions.

Intersection: A vehicle is allowed to cross the intersection according to the direction of travel when a traffic signal is green.

Multi-lane: A vehicle can move to parallel lanes. If the direction of travel is a right-turn, the vehicle moves to a right-turn exclusive lane.

\section{3) Search of Traffic Signals by Using the H-GA-PSO Algorithm}

A fast algorithm is necessary to achieve real-time control for traffic control because the processing of the prediction and search to decide on the traffic signals of $k$-th cycle has to finish within a minimum time $t_{\text {minimum }}^{l}$ of the green-signal as shown in Fig. 4. In a global version of the PSO method, all particles tend to gbest; therefore, particles may fall into a local minimum point easily, as illustrated in Fig. 24, when the particle size or searching time is limited to realize realtime control.

Among the modified PSO methods are the Linearly Decreasing Inertia Weight Method (LDIWM) [30] and the Constriction Factor Method (CFM) [31]. The LDIWM method makes the coefficient $w$ of particle velocity Eq. (7) smaller with an increase in the number of searches shown in Eq. (15), where $g$ is the number of a search and $G$ is the maximum value of $g$. In addition, the CFM method makes the coefficients of particle velocity greater compared with the coefficient of terms for pbest and gbest.

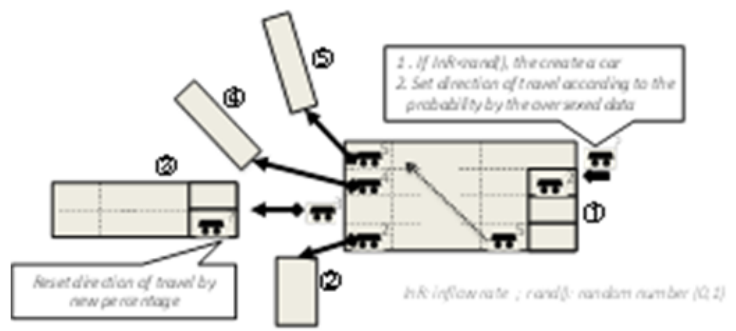

Fig. 23. Rules of vehicle movements for cellular automaton microsimulator at r-way intersection.

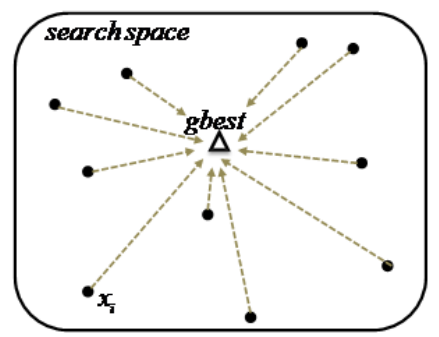

Fig. 24. Convergence of global-version particle swarm optimization. 
These methods are expected to produce an improvement in the search; however, it is also possible for the particle to fall into a local minimum point when the particle size is small.

$$
\begin{aligned}
& w=w_{\max }-\left(w_{\max }-\mathrm{w}_{\min }\right) \cdot \frac{g}{G}, \\
& v_{i}^{m+1}=\chi \cdot\left[v_{i}^{m}+c_{1} \cdot \text { rand }() \cdot\left(\text { pbest }_{i}^{m}-x_{i}^{m}\right)\right. \\
& \left.+c_{2} \cdot \operatorname{rand}() \cdot\left(\text { gbest }^{m}-x_{i}^{m}\right)\right] \\
& x_{i}^{m+1}=x_{i}^{m}+v_{i}^{m+1} \text {, } \\
& \chi=\frac{2}{\left|2-\varphi-\sqrt{\varphi^{2}-4 \varphi}\right|}, \\
& \varphi=c_{1}+c_{2} \text {. }
\end{aligned}
$$

A hierarchical PSO (H-PSO, local version) method [32] can overcome this local minimum problem. The H-PSO method divides particles into several groups, and then the best particle in each group is located in a high hierarchy and the others are in a low hierarchy, illustrated in Fig. 25.

$$
\begin{aligned}
v_{i j}^{m+1}= & w_{l} \cdot v_{i j}^{m}+c_{l l} \cdot \operatorname{rand}() \cdot\left(\text { pbest }_{i j}^{m}-x_{i j}^{m}\right), \\
& +c_{l 2} \cdot \operatorname{rand}() \cdot\left(\text { lbest }_{i}^{m}-x_{i j}^{m}\right) \\
x_{i j}^{m+1}= & x_{i j}^{m}+v_{i j}^{m+1}, \\
v_{i}^{m+1}= & \left.w_{h} \cdot v_{i}^{m}+c_{h 1} \cdot \operatorname{rand}\right) \cdot\left(\text { pbest }_{i}^{m}-x_{i}^{m}\right), \\
& \left.+c_{h 2} \cdot \text { rand }_{0}\right) \cdot\left(\text { gbest }^{m}-x_{i}^{m}\right) \\
x_{i}^{m+1}= & x_{i}^{m}+v_{i}^{m+1} .
\end{aligned}
$$

In the H-PSO method, the particles in the low hierarchy are updated using Eqs. (20) and (21), and the particles in the high hierarchy are updated using Eqs. (22) and (23), respectively. In Eq. (20), lbest is a position with the best fitness in each group. This method, however, has the defect of slow convergence. The H-PSO method is newly modified to achieve higher convergence as the term of gbest is added to Eq. (7) of the particle velocity for the groups in the low hierarchy as shown in Eq. (24).

$$
\begin{aligned}
& v_{i j}^{m+1}=w_{l} \cdot v_{i j}^{m}+c_{l 1} \cdot \operatorname{rand}\left(0 \cdot\left(\text { pbest }_{i j}^{m}-x_{i j}^{m}\right)\right. \\
& \quad+c_{l 2} \cdot \operatorname{rand}() \cdot\left(\text { lbest } t_{i}^{m}-x_{i j}^{m}\right)+c_{l 3} \cdot \operatorname{rand}() \cdot\left(\text { gbest }^{m}-x_{i j}^{m}\right)
\end{aligned}
$$

At the same time, a genetic algorithm (GA) is newly introduced to update a selected particle position to avoid the local minimum problem. The number of chromosomes is 21 ; one chromosome represents one cycle of the traffic signal, and one gene is an integer from 4 to 9 seconds. An example of a chromosome is illustrated in Fig. 26. Crossover processing is used to exchange two particles between two groups, and mutation processing is used to reset a particle around gbest or lbest, illustrated in Fig. 27.

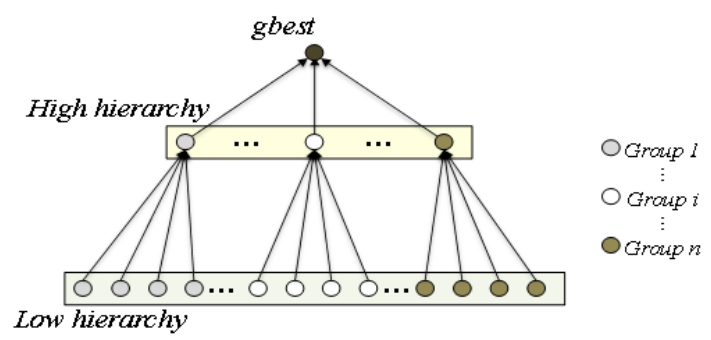

Fig. 25. Structure of hierarchical particle swarm optimization.

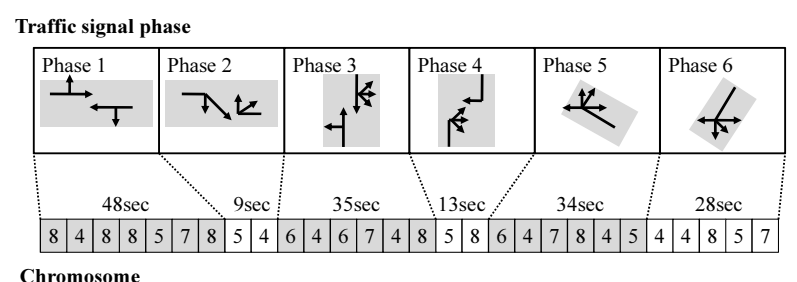

Fig. 26. Example of a chromosome.

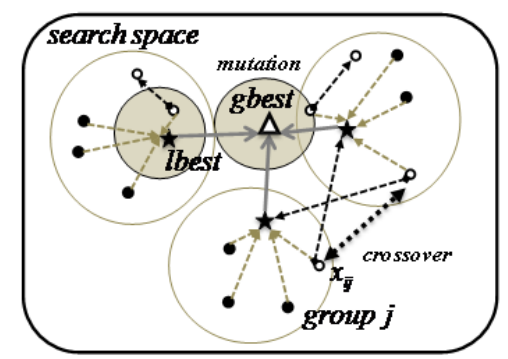

Fig. 27. Genetic algorithm of updating for position of particles.

Furthermore, a rule for the initial generation of particles is newly introduced to generate the next further particle for a distance L away from a particle as illustrated in Fig. 28. The procedure for the new H-GA-PSO algorithm, which combines H-PSO with the modified velocities, GA operations, and the initial generation of particles, is shown in Fig. 29.

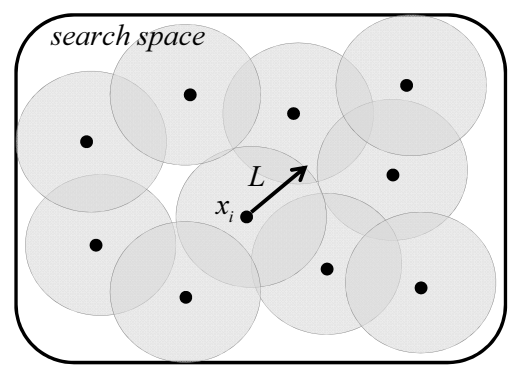

Fig. 28. Initial generation of particles. 


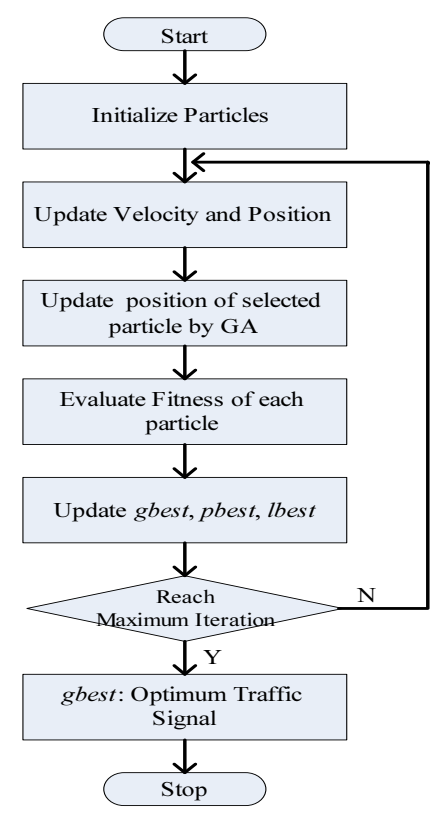

Fig. 29. Procedure of H-GA-PSO algorithm. H: hierarchical, GA: genetic algorithm, PSO: particle swarm optimization.

\section{Simulation}

A simulation was carried out to confirm the effectiveness of the real-time stochastic optimum control of traffic signals at an $r$-way intersection based on the H-GA-PSO method using actual traffic flow data. The actual data was measured on 15 September 2010, 7:30-8:30 AM at Ijiri 6-way intersection, Fukuoka, Japan, as shown in Fig. 30, and a frame of the CA traffic micro-simulator is illustrated in Fig. 31. The phases of the traffic signals, conditions of traffic flows, and parameters of the CA micro-simulator based on the actual measured data are shown in Tables 2 and 3 , respectively. Therefore, the minimum time $t_{\text {minimum }}^{l}$ of the green-signal was determined to be 30 seconds. Table 4 shows the parameters of fitness function Eq. (13).

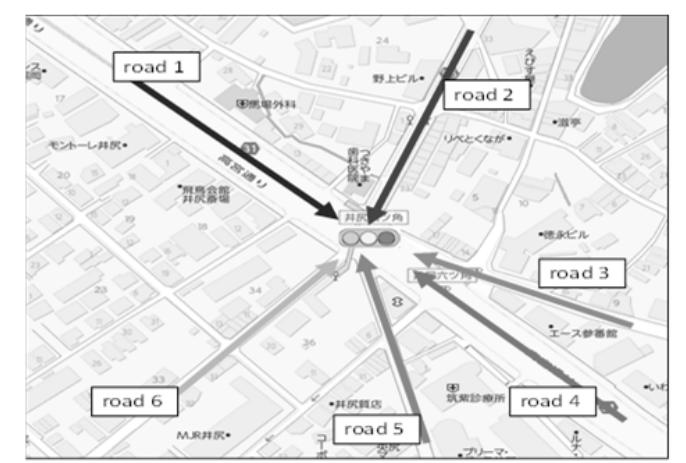

Fig. 30. Map of ljiri 6-way intersection.

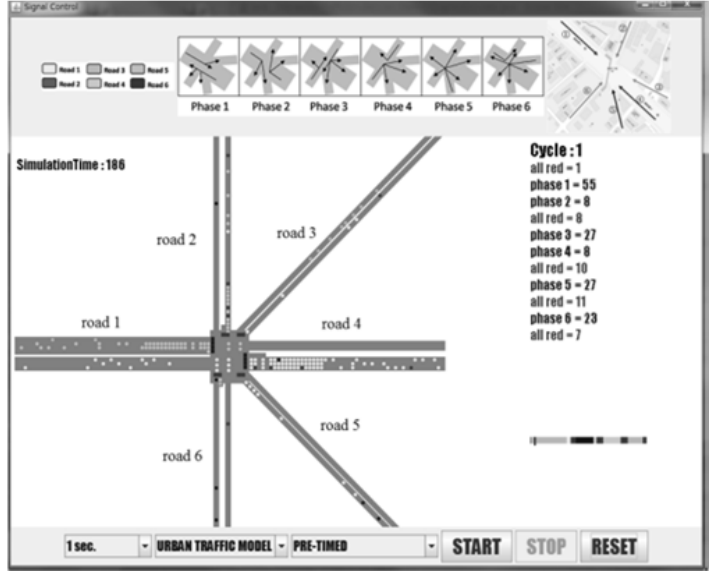

Fig. 31. A frame of cellular automaton traffic micro-simulator.

Table 2. Traffic signals of pretimed control at ljiri 6-way intersection

\begin{tabular}{|c|c|c|c|c|c|c|}
\hline \multirow[t]{2}{*}{$\begin{array}{l}\text { No. of } \\
\text { Phase }\end{array}$} & & 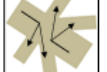 & 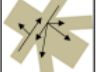 & $+V$ & & $* 1$ \\
\hline & Phase 1 & Phase 2 & Phase 3 & Phase 4 & Phase 5 & Phase 6 \\
\hline Time (sec) & 55 & 8 & 27 & 8 & 27 & 23 \\
\hline $\begin{array}{l}\text { Adjustable } \\
\text { range }\end{array}$ & $30-80$ & $4-12$ & $15-35$ & $4-12$ & $15-35$ & $15-35$ \\
\hline
\end{tabular}

Table 3. Parameters of micro-simulator

\begin{tabular}{lcccccc}
\hline \multicolumn{1}{c}{ Road } & 1 & 2 & 3 & 4 & 5 & 6 \\
\hline Length (cells) & 40 & 40 & 50 & 35 & 40 & 50 \\
Lane number & 4 & 2 & 1 & 4 & 1 & 2 \\
Traffic flow & $800-$ & $250-$ & $260-$ & $1200-$ & $150-$ & $120-$ \\
(pcu/h) & 1000 & 290 & 340 & 1400 & 170 & 140 \\
\hline
\end{tabular}

Table 4. Parameters of fitness function

\begin{tabular}{lcccccc}
\hline \multicolumn{1}{c}{ Road } & 1 & 2 & 3 & 4 & 5 & 6 \\
\hline$w_{h}(\%)$ & 20 & 16 & 18 & 24 & 12 & 10 \\
$q^{\max }($ cells $)$ & 25 & 25 & 30 & 20 & 25 & 30 \\
\hline
\end{tabular}

In addition, the parameters of the H-GA-PSO algorithm are as follows: $w_{h}=0.729, w_{i}=0.5, c_{h l}=c_{h 2}=1.49, c_{i l}=1$, $c_{i 2}=1, c_{i 3}=0.5$, particle size is 21 , the number of groups is 3 , and the maximum iteration is 25 .

For an examination of traffic signal controls, a simulation was carried out using each of the GA, CFM, LDIWM, HPSO, and H-GA-PSO algorithms. There are three traffic congestion levels (normal, high, and very high), and their results from 20 times are shown in Table 5.

The convergence of fitness in the case of very high congestion, and the mean fitness values and their standard deviations are shown in Figs. 32 and 33, respectively. 
Table 5. Comparison of traffic signal controls

\begin{tabular}{|c|c|c|c|c|c|c|c|c|c|c|c|c|}
\hline \multirow{3}{*}{ Method } & \multicolumn{12}{|c|}{ Traffic congestion } \\
\hline & \multicolumn{4}{|c|}{ Normal } & \multicolumn{4}{|c|}{ High } & \multicolumn{4}{|c|}{ Very High } \\
\hline & $\begin{array}{l}\text { Mean } \\
\text { fitness }\end{array}$ & $\begin{array}{c}\text { Best } \\
\text { fitness }\end{array}$ & $\begin{array}{l}\text { Worst } \\
\text { fitness }\end{array}$ & $\begin{array}{l}\text { Standard } \\
\text { deviation }\end{array}$ & $\begin{array}{l}\text { Mean } \\
\text { fitness }\end{array}$ & $\begin{array}{c}\text { Best } \\
\text { fitness }\end{array}$ & $\begin{array}{l}\text { Worst } \\
\text { fitness }\end{array}$ & $\begin{array}{l}\text { Standard } \\
\text { deviation }\end{array}$ & $\begin{array}{l}\text { Mean } \\
\text { fitness }\end{array}$ & $\begin{array}{l}\text { Best } \\
\text { fitness }\end{array}$ & $\begin{array}{l}\text { Worst } \\
\text { fitness }\end{array}$ & $\begin{array}{r}\text { Standard } \\
\text { deviation }\end{array}$ \\
\hline GA & 1.64 & 1.49 & 1.89 & 0.13 & 2.53 & 2.41 & 3.00 & 0.15 & 7.81 & 7.62 & 7.95 & 0.06 \\
\hline CFM & 1.41 & 1.38 & 1.52 & 0.04 & 2.44 & 2.37 & 2.80 & 0.13 & 7.69 & 7.61 & 7.79 & 0.08 \\
\hline LDIWM & 1.47 & 1.38 & 1.79 & 0.14 & 2.42 & 2.37 & 2.59 & 0.06 & 7.69 & 7.61 & 7.79 & 0.08 \\
\hline H-PSO & 1.52 & 1.37 & 1.79 & 0.11 & 2.43 & 2.37 & 2.62 & 0.06 & 7.71 & 7.61 & 7.80 & 0.08 \\
\hline H-GA-PSO & 1.38 & 1.36 & 1.40 & 0.01 & 2.39 & 2.37 & 2.45 & 0.03 & 7.62 & 7.61 & 7.67 & 0.02 \\
\hline
\end{tabular}

GA: genetic algorithm, CFM: constriction factor method, LDIWM: linearly decreasing inertia weight method, H-PSO: hierarchical particle swarm optimization.

The searches by the GA and H-PSO methods do not reach the minimum value by a limited number of searches, and the searches by the LDIWM and CFM methods might fall into a local minimum point. Moreover, the mean of the traffic queue length, total delay time, and velocity are shown in Figs. 34, 35, and 36, respectively.

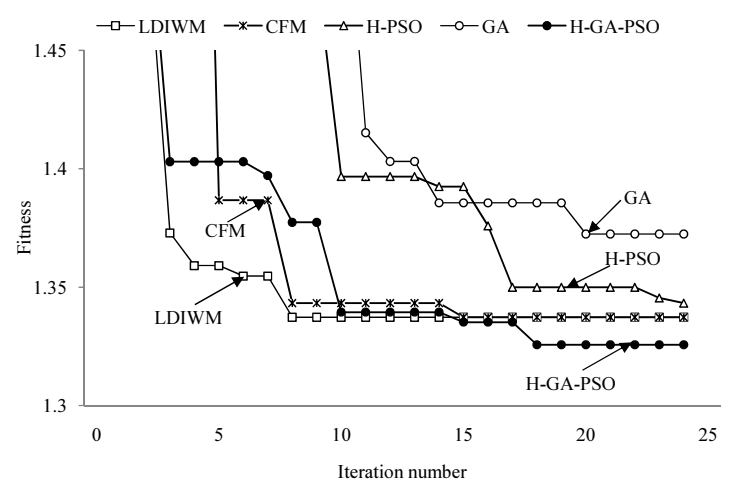

Fig. 32. Convergence of fitness in the case of very high congestion. GA: genetic algorithm, CFM: constriction factor method, LDIWM: linearly decreasing inertia weight method, H-PSO: hierarchical particle swarm optimization.

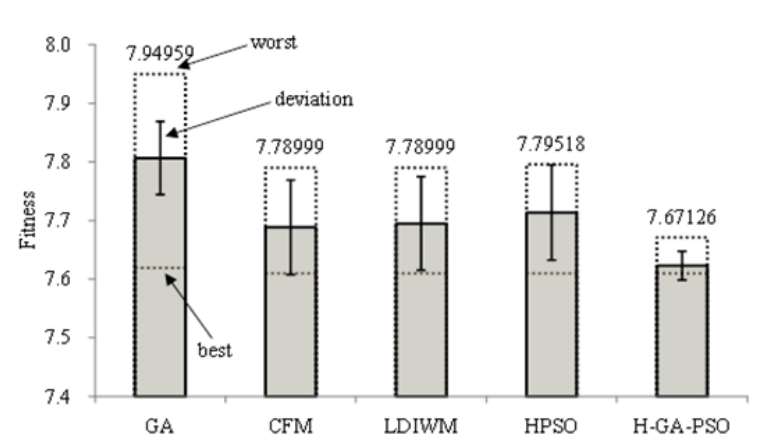

Fig. 33. Mean fitness and standard deviation of each method. GA: genetic algorithm, CFM: constriction factor method, LDIWM: linearly decreasing inertia weight method, H-PSO: hierarchical particle swarm optimization.
The mean traffic queue by the H-GA-PSO control was reduced by $15(\%)$ compared with the pretimed control, and the total delay time was reduced by $20(\%)$ and $6(\%)$ compared with the pretimed and CFM methods, respectively. In addition, the mean velocity was accelerated $1.5 \%$ and $0.5 \%$ compared with the pretimed and CFM methods, respectively.

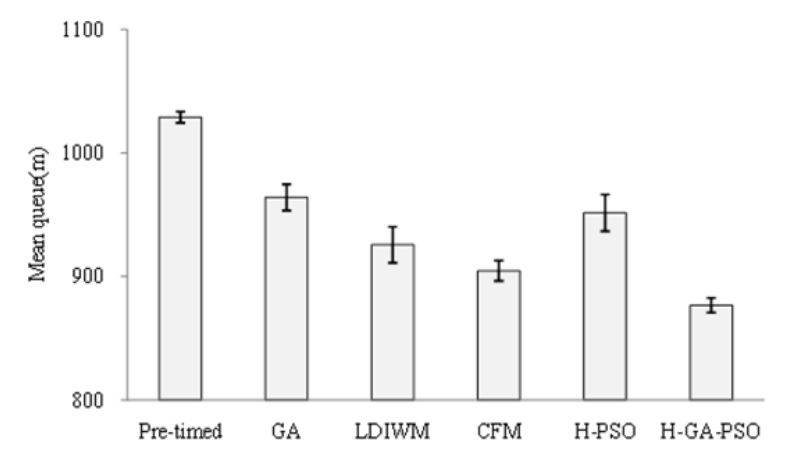

Fig. 34. Mean traffic queue length (m). GA: genetic algorithm, CFM: constriction factor method, LDIWM: linearly decreasing inertia weight method, H-PSO: hierarchical particle swarm optimization.

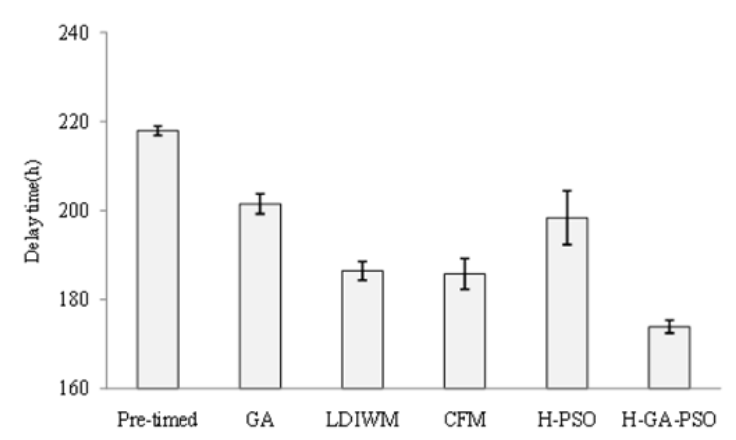

Fig. 35. Total delay time (hr). GA: genetic algorithm, CFM: constriction factor method, LDIWM: linearly decreasing inertia weight method, H-PSO: hierarchical particle swarm optimization. 


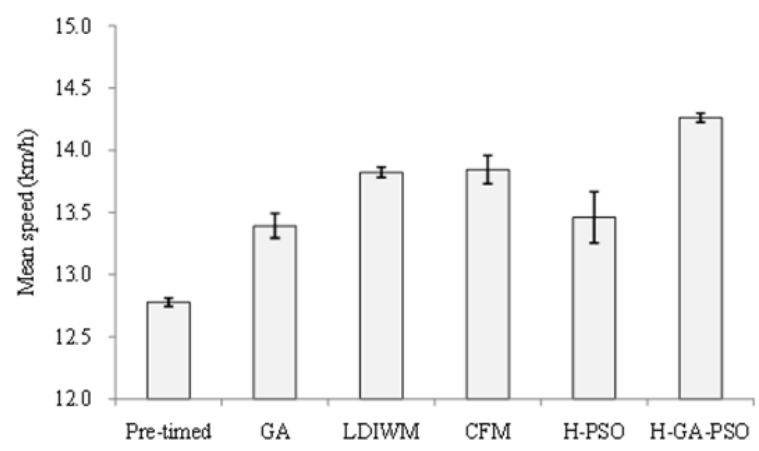

Fig. 36. Mean velocity. GA: genetic algorithm, CFM: constriction factor method, LDIWM: linearly decreasing inertia weight method, H-PSO: hierarchical particle swarm optimization.

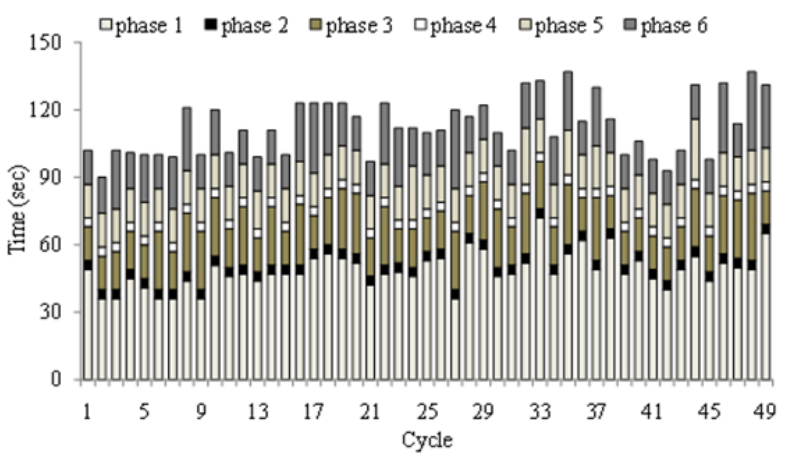

Fig. 37. Traffic signals at ljiri 6-way intersection by H-GA-PSO control. $\mathrm{H}$ : hierarchical, GA: genetic algorithm, PSO: particle swarm optimization.

Finally, the traffic signals at Ijiri 6-way intersection of Fig. 30 by the H-GA-PSO algorithm are shown in Fig. 37.

\section{CONCLUSIONS}

Traffic congestion is a serious problem in modern cities, and traffic signal control is an effective method for reducing traffic jams. For actual random traffic flows, a stochastic model of traffic flows and traffic jams was constructed by using a $\mathrm{BN}$, and then the probabilistic distributions of traffic jams were predicted by using a BN stochastic model and a cellular automaton model. In addition, a new H-GA-PSO algorithm was used to search for the optimum traffic signals of crossroads and multi-way intersections to realize realtime control. The effectiveness of the real-time stochastic optimum control of traffic signals was confirmed by simulations based on actual traffic data.

\section{REFERENCES}

[1] C. Peck, P. T. W. Gorton, and D. Liren, "The application of SCOOT in developing countries," in Proceedings of the 3rd International Conference on Road Traffic Control, London, pp. 104-109, 1990.

[2] A. G. Sims and K. W. Dobinson, "The Sydney coordinated adaptive traffic (SCAT) system philosophy and benefits," IEEE Transactions on Vehicular Technology, vol. 29, no. 2, pp. 130-137, 1980.

[3] N. H. Gartner, "OPAC: a demand-responsive strategy for traffic signal control," Transportation Research Record, no. 906, pp. 75-81, 1983.

[4] A. Kouvelas, K. Aboudolas, M. Papageorgiou, and E. B. Kosmatopoulos, "A hybrid strategy for real-time traffic signal control of urban road networks," IEEE Transactions on Intelligent Transportation Systems, vol. 12, no. 3, pp. 884-894, 2011.

[5] J. Lee, B. Abdulhai, A. Shalaby, and E. H. Chung, "Real-time optimization for adaptive traffic signal control using genetic algorithms," Journal of Intelligent Transportation Systems, vol. 9, no. 3, pp. 111-122, 2005.

[6] C. Dong, S. Huang, and X. Liu, "Urban area traffic signal timing optimization based on Sa-PSO," in Proceedings of the International Conference on Artificial Intelligence and Computational Intelligence, Sanya, China, pp. 80-84, 2010.

[7] Y. S. Murat and E. Gedizlioglu, "A fuzzy logic multi-phased signal control model for isolated junctions," Transportation Research Part C: Emerging Technologies, vol. 13, no. 1, pp. 19-36, 2005.

[8] Y. J. Cao, N. Ireson, L. Bull, and R. Miles, "Design of a traffic junction controller using classifier system and fuzzy logic," in Proceedings of the 6th International Conference on Computational Intelligence, Theory and Applications: Fuzzy Days, Dortmund, Germany, pp. 342-353, 1999.

[9] B. P. Gokulan and D. Srinivasan, "Distributed geometric fuzzy multiagent urban traffic signal control," IEEE Transactions on Intelligent Transportation systems, vol. 11, no. 3, pp. 714-727, 2010.

[10] J. Niittymaki and V. Kononen, "Traffic signal controller based on fuzzy logic," in Proceedings of IEEE International Conference on Systems, Man, and Cybernetics, Nashville: TN, vol. 5, pp. 35783581, 2000.

[11] J. Qiao, N. Yang, and J. Gao, "Two-stage fuzzy logic controller for signalized intersection," IEEE Transactions on System, Man, and Cybernetics, Part A: Systems and Humans, vol. 41, no. 1, pp. 178184, 2011.

[12] C. Kim and Y. S. Hong, "Traffic signal using smart agent system," American Journal of Applied Sciences, vol. 5, no. 11, pp. 14871493, 2008.

[13] L. Zang, L. Jia, and Y. Luo, "An intelligent control method for urban traffic signal based on fuzzy neural network," in Proceedings of the 6th World Congress on Intelligent Control and Automation, Dalian, China, pp. 3430-3434, 2006.

[14] H. Adeli and A. Samant, "An adaptive conjugate gradient neural network-wavelet model for traffic incident detection," ComputerAided Civil and Infrastructure Engineering, vol. 15, no. 4, pp. 
251-260, 2000.

[15] K. H. Chao, R. H. Lee, and M. H. Wang, "An intelligent traffic light control based on extension neural network," in Proceedings of the 12th International Conference on Knowledge-Based Intelligent Information and Engineering Systems, Zagreb, Croatia, pp. 17-24, 2008.

[16] M. C. Choy, D. Srinivasan, and R. L. Cheu, "Neural networks for continuous online learning and control," IEEE Transactions on Neural Networks, vol. 17, no. 6, pp. 1511-1531, 2006.

[17] D. Srinivasan, M. C. Choy, and R. L. Cheu, "Neural networks for real-time traffic signal control," IEEE Transactions on Intelligent Transportation Systems, vol. 7, no. 3, pp. 261-272, 2006.

[18] K. Wen, W. Yang, and S. Qu, “A stochastic adaptive traffic signal control model based on fuzzy reinforcement learning," in Proceedings of the 2nd International Conference on Computer and Automation Engineering, Singapore, pp. 467-465, 2010.

[19] S. Lu, X. Liu, and S. Dai, “Adaptive and coordinated traffic signal control based on Q-learning and multiband model," in Proceedings of IEEE Conference on Cybernetics and Intelligent Systems, Chengdu, China, pp. 765-770, 2008.

[20] L. Kuyer, S. Whiteson, B. Bakker, and N. Vlassis, "Multiagent reinforcement learning for urban traffic control using coordination graphs," in Proceedings of the 19th European Conference on Machine Learning and Knowledge Discovery in Database, Antwerp, Belgium, pp. 656-671, 2008.

[21] E. Bingham, "Reinforcement learning in neurofuzzy traffic signal control," European Journal of Operational Research, vol. 131, no. 2, pp. 232-241, 2001.

[22] I. Arel, C. Liu, T. Urbanik, and A. G. Kohls, "Reinforcement learning-based multi-agent system for network traffic signal control," IET Intelligent Transportation Systems, vol. 4, no. 2, pp. 128-135, 2010.

[23] P. G. Balaji, X. German, and D. Srinivasan, "Urban traffic signal control using reinforcement learning agents," IET Intelligent
Transportation Systems, vol. 4, no. 3, pp. 177-188, 2010.

[24] R. E. Neapolitan, Learning Bayesian Networks, Upper Saddle, NJ: Pearson Prentice Hall, 2004.

[25] C. Y. Cui, J. S. Shin, M. Miyazaki, and H. H. Lee, "Real-time traffic signal control for optimization of traffic jam probability," IEEJ Transactions on Electrics, Information and Systems, vol. 132, no. 1, pp. 21-31, 2012.

[26] C. Y. Cui, J. S. Shin, and H. H. Lee, "Real-time traffic signal learning control using BPNN based on predictions for probabilistic distribution of standing vehicles," Journal of Artificial Life and Robotics, vol. 15, no. 1, pp. 58-61, 2010.

[27] K. Nagel and M. Schreckenberg, "A cellular automaton model for freeway traffic," Journal de Physique I, Vol. 2, No. 12, pp. 2221$2229,1992$.

[28] J. Kennedy and R. Eberhart, "Particle swarm optimization," in Proceedings of IEEE International Conference on Neural Networks, Perth: WA, pp. 1942-1948, 1995.

[29] C. Y. Cui, G. Y. Han, M. Miyazaki, and H. H. Lee, "Real-time traffic signal control of multi-way intersection using H-GA-PSO algorithm based on optimization of traffic jam probability," IEEJ Transactions on Electrics, Information and Systems, vol. 132, no. 12, pp. 1982-1991, 2012.

[30] Y. Shi and R. Eberhart, “A modified particles swarm optimizer," in Proceedings of the IEEE World Congress on Computational Intelligence, Anchorage: AK, pp. 69-73, 1998.

[31] M. Clerc and J. Kennedy, "The particle swarm - explosion, stability, and convergence in a multidimensional complex space," IEEE Transactions on Evolutionary Computation, vol. 6, no. 1, pp. 58-73, 2002.

[32] Janson S., Middendorf M.: "A hierarchical particle swarm optimizer and its adaptive variant," IEEE Transactions on Systems, Man, and Cybernetics, Part B: Cybernetics, vol. 35, no. 6, pp. 1272-1282, 2005.

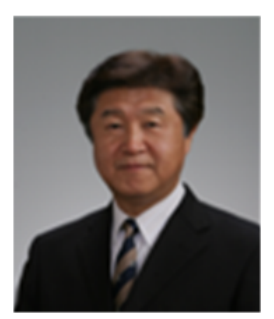

\section{Hee-Hyol Lee}

received the Ph.D. degree from the Graduate School of Science and Engineering of Waseda University in 1986. After his time as an associate professor and a professor at Fukuoka Institute of Technology, he became a professor at Waseda University in 2003 and has continued until the present. He was a visiting professor in the Department of Electrical Engineering of the University of California, Los Angeles (UCLA), USA, from 2011 to 2012. He is currently engaging in research in the area of analysis, estimation, and control of stochastic systems, voice and character recognition, sliding mode control, decoupled control, power generation control, and intelligent control. He was awarded the Sawaragi Memorial Award of the Institute of System, Control and Information Engineers in 1986 and the 30th Anniversary Memorial Award for an Excellent Paper of IEEJ Transactions in 2003. He is a member of SICE, ISCI, IEEJ, and IEEE. 